\title{
Developing a consequence management assessment framework
}

\author{
Kenneth McDonald* \\ Department of Systems Engineering, \\ United States Military Academy, \\ 10996 West Point, New York, USA \\ Email: kenneth.mcdonald@usma.edu \\ *Corresponding author
}

\section{Robert Prins}

Nuclear Science and Engineering Research Center, United States Military Academy, 10996 West Point, New York, USA Email: rprins@ara.com

\begin{abstract}
This research developed an assessment process framework and tool to assess national consequence management capacity. The research applied a value focused thinking and the Systems Design Process (SDP) to create a framework that effectively analyses a nation's capacity to handle extreme crisis situations to include Chemical, Biological, Radiological, Nuclear and Explosive (CBRNE) events. The research conducted extensive stakeholder analysis to identify critical metrics in identifying a nation's capacity and capabilities. Weighting and scoring of the value metrics was applied to develop scoring assessments. The results provided a usable assessment model that tracks capacity progress over time. The model provides a value contribution to the development of an effective assessment tool and achieves the goal of providing a practical tool for assessment. This research takes assessing a very difficult and complex system and makes it relatively easy and simple, while providing solid evidence of capacity improvement or deterioration over time.
\end{abstract}

Keywords: environmental disasters; consequence management; value focused thinking; environmental health; prophylaxis response; CBRNE; chemical, biological, radiological, nuclear and explosive events.

Reference to this paper should be made as follows: McDonald, $\mathrm{K}$. and Prins, R. (2019) 'Developing a consequence management assessment framework', Int. J. Nuclear Safety and Security, Vol. 1, No. 1, pp.53-63.

Biographical notes: Kenneth McDonald is currently a Professor of Engineering Management, Department of Systems Engineering, United States Military Academy, West Point. He is also the Director of the Centre for Nation Reconstruction and Capacity Development. He has worked in a number of academic fields which has provided him a unique perspective of combing both the practice of Engineering and Culture. His research and consulting interests are in capacity development and decision analysis as applied to infrastructure and other complex enterprises especially in the arena of reconstruction and capacity development. 
Robert Prins was the Director of the Nuclear Science and Engineering Research Centre, United States Military Academy. He has taught Nuclear Physics as an Assistant professor, Department of Physics and Nuclear Engineering, West Point. His current research interests include megacity impact of natural and manmade disasters.

This paper is a revised and expanded version of a paper entitled 'Assessing Consequence Management' presented at the 'General Donald R. Keith Memorial Capstone Conference', 2 May 2013.

\section{Introduction}

In this very complex and dangerous world, nations are faced with greater and greater threats from all sorts and types of environmental and manmade disasters. The intensity of environmental events is seemly becoming far greater. Likewise, one of the predominatnt manmade threats comes from Chemical, Biological, Radiological, Nuclear and High Explosive (CBRNE) weapon systems. With these potential threats becoming a greater reality for all nations, the need for an adequate response to such events is more critical. Indeed, the inability of a nation to adequately respond will place sitting governments in a very precarious position. This research addresses part of the consequence management challenge facing nations today: assessment. The purpose of this research is to develop a framework to assess and evaluate the capabilities and capacities for a nation's consequence management program. This framework tracks improvements and identifies weakness and allows a nation to focus resources and money into areas that require immediate assistance.

There are limited metric assessment systems that provide meaningful analysis to show definitive improvement in measured areas of consequence management. Some of the challenges include automation, subjectiveness, numerous metrics, untrained personnel, etc. Most assessments are done by hand or are placed in pdf files and stored thus limiting the ability of assessors to access historical data to draw trends. In some cases, there are agencies with hundreds of metrics making it exceedingly difficult to track progress and standardise the collection process. In most cases there is not an apparent calibrated training program for personnel collecting the input data. This lack of proper training combined with a large range of metrics produces an inaccurate picture of the strengths and weaknesses of a nation's consequence management program.

There is extensive literature on consequence management from an environmental disaster perspective and some nations have developed tremendous response capabilities. However, there is room for improvement as populations grow and megacities expand. The result of this growth is greater detrimental impact that an environmental disaster can have on national population centers. Consequence management after environmental disasters requires extensive coordinated effort by numerous government and non-government agencies. The key elements of environmental disaster consequence management are numerous and complex with each element interacting and effecting each other in ways very difficult to understand. There is a demand to ensure that efficiency and effectiveness is at levels that will provide a population the needed relief. For example, one area highlighted in research is the need for information throughout every phase of disaster management (Guh-Sapir and Lechat, 1986). Without good information 
flow and control disasters expand and grow with the result of more injury and loss of life. The literature has a host of social research into the areas of environmental disaster response; however, there is less focus on more systems analysis approaches to this topic. Disasters are large intractable problems that test the ability of nations to protect their people and community infrastructure. How local communities, cities and nations interact and operate in conjunction with one another has not been completely studied. There is a lack of detailed analysis to provide a general understanding of the current systems, and the operational gaps. There is no coherent process to develop capacities to overcome these shortfalls (Altay and Green III, 2006).

Consequence management also encompasses the response to disasters or catastrophic events that are manmade. In the realm of Weapons of Mass Destruction (WMDs), consequence management is defined as "...a process to mitigate the effects of the use of weapons of mass destruction..." to include CBRNE events (Bennett and Love, 2004). One of the primary concerns of consequence management in this realm is assessing a nation's ability to plan and execute an incident response that is composed of several different agencies. Since the Cold War, WMDs have been at the forefront of foreign policies and a constant source of international tension (Taylor et al., 1999). While the threat of a catastrophic exchange of nuclear weapons has diminished over the years, the threat of CBRNE events and WMDs has risen. A policy of deterrence cannot effectively stop all enemies and terrorist organisations from using these technologies to inflict mass casualties upon nations (Betts, 2012). In particular, the threat of CBRNE attacks by rogue nations and terrorist groups have increased dramatically, thus causing the international community to devote more attention to consequence management (Bennett and Love, 2004).

With the growing demand for nuclear technology for non-military purposes, the need to measure a nation's consequence management capabilities has gained greater significance. The increasing access of an organisation bent on destabilising a city or nation to more lethal weapons makes the need for effective response to these types of attacks more significant. The ability of a nation or a group of nations to effectively measure their consequence management programs is of upmost importance not only from a preparation perspective but also from a population protection perspective. For example, the increase in nuclear activity in North Korea is a threat to the USA and its allies within the Asian-Pacific region (Arms Control Association, 2014). By developing the capacity and capabilities of the partner nations in that area, the USA is able to gain a stronger position and a greater defense against this looming threat.

The literature on consequence management is robust but is still somewhat limited when the aperture is broadened to include both environmental and manmade disasters. There is limited, if any, literature on assessing national operational consequence management capabilities. As such, this research is an attempt to fill in some of the gaps that remain unchallenged in this very complex and needed area.

\section{Methodology}

This research focused on producing a framework to collect assessment data and turning it into quantitative data by using 26 value measures loosely based off of response criteria developed by the Department of Homeland Security, through a multiple objective decision analysis model. The research was complicated by the limited information 
concerning the most critical aspects of consequence management. In other words, what are those essential components necessary to have a good consequence management response? In other to tackle this challenge an effective and efficient methodology was employed to provide a construct to conduct the research.

\subsection{Systems decision process}

Making decisions in a highly complex world requires a deep level of insight and understanding about a system that demands understanding of how systems work and interact. It is critically important for any decision maker to be able to assess second and third order effects before allocation of critical resources. The Systems Decision Process (SDP) is an effective tool for decision makers to exhaustively analyse their problem, consider alternatives and make informed decisions. The SDP is a collaborative, iterative, and value-based decision process that can be applied in any system life cycle stage (Parnell et al., 2010). The SDP's holistic framework provides decision makers with the ability to use it to analyse any system. Using the SDP helps understand the status quo, the desired end state and the path needed to get to the end state. It is conducted in four phases that starting with the identification of the problem and ending with the systems retirement (see Figure 1).

Figure 1 The systems decision process (Parnell et al, 2010)

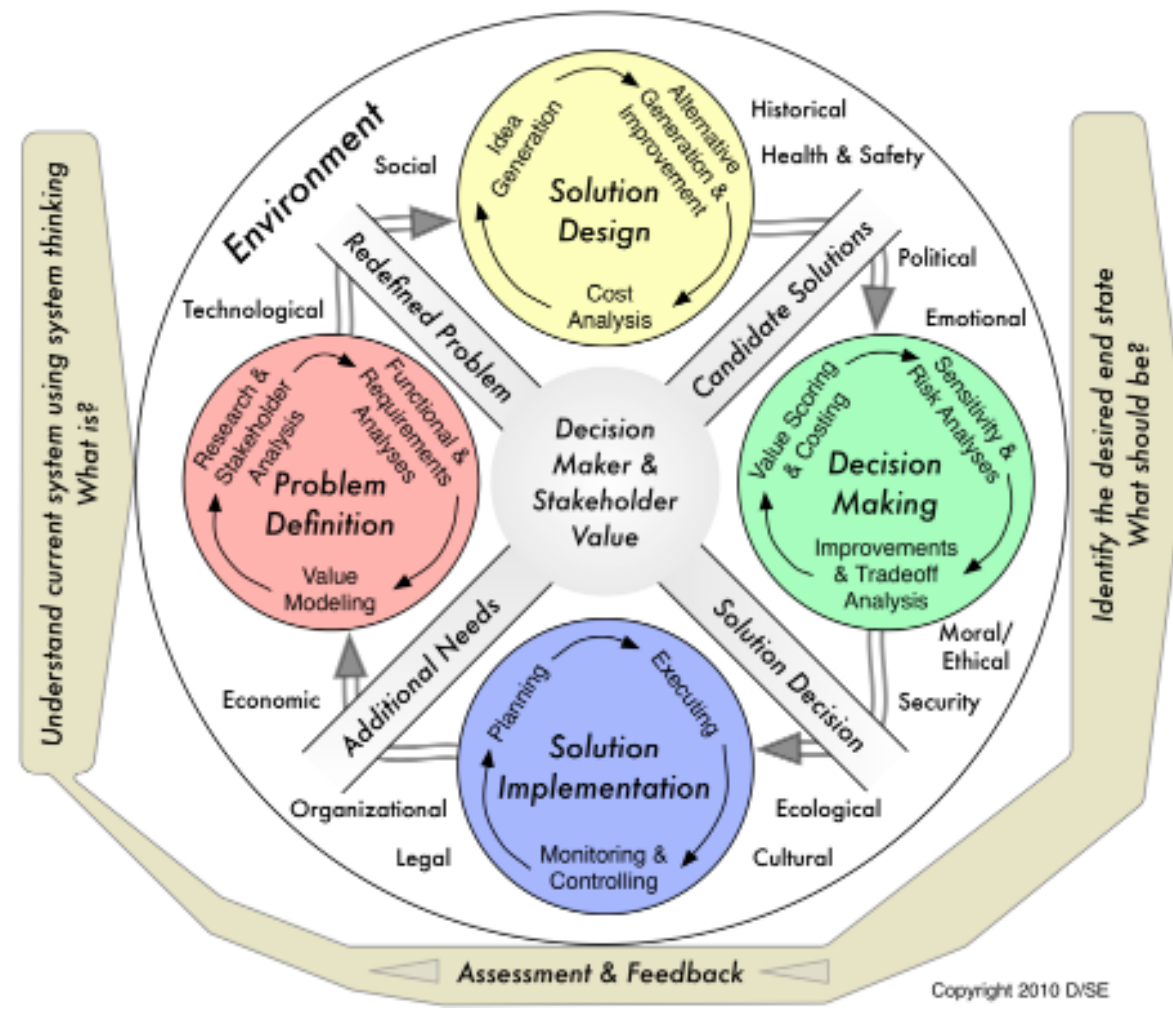


The SDP begins with the problem definition phase. The goal of this phase is to define a clear problem statement. Often times the initial problem is never the real problem (Parnell et al., 2010). A clear problem statement is achieved primarily through extensive research and stakeholder analysis. During this phase the systems engineer gathers as much information as possible about the system. This process includes research and stakeholder analysis, functional and requirements analysis and value modeling. While research is invaluable throughout the entire SDP, it is particularly useful early on in order to identify functions, objectives, and constraints.

The second phase is solution design, which involves developing candidate solutions to present to the stakeholder. The candidate solutions must meet the needs, wants and desires that were identified during the problem definition phase. The goal is to present the decision maker with more than one solution so that they can assess candidate solutions. The SDP provides different qualitative and quantitative value models that demonstrate solutions specific strengths and weaknesses. At the end the decision maker should have all of the information about the potential solutions and be ready to make a decision on which solution to select.

The decision making phase is where the systems engineer makes a recommendation to the decision maker. The tasks that the systems engineer must accomplish during this phase are: score and cost the candidate solutions, conduct sensitivity and risk analyses, use value-focused thinking to improve solutions, and apply tradeoff analysis to compare value versus cost associated with candidate solutions (Parnell et al, 2010). Then the systems engineer completes the analysis and a solution is selected.

Solution implementation is potentially the most difficult phase to accomplish. Implementation needs to be a consideration during the entirety of the SDP because implementation may be a deciding factor when choosing a solution. This phase is extremely important because it involves turning the stakeholder's vision into a reality. The physical development of the project happens during this phase with the end result being the solution brought to life with a plan for maintenance until it reaches the end of its life cycle.

\section{Model development}

The Capacity Assessment Model (CAM) is a spreadsheet-based value scoring model that transforms qualitative data collected by trained personnel into quantitative data that can be understood by stakeholders and users. Quantitative data from the model can be used to track a nation's capacity development. The data is archived in order to track progress over time. Likewise, weighting and value measure scoring can be modified as needed. The value of the CAM is its simplicity and clarity.

\subsection{Research and stakeholder analysis}

The problem definition phase required an extensive amount of time to gather and analyse information on consequence management. Through a detailed literature review, use of systemigrams and numerous interviews, data was compiled into a findings, conclusion, and recommendations table. This table technique provides the researcher to synthesise and bundle like facts together to draw conclusions. Once the conclusions are completed, recommendations are determined which will help address those conclusions. From 
these recommendations a fundamental objective is determined which is the one objective required for the system to solve the stakeholder challenge. In the case of consequence management, the fundamental objective was determined to be: "Measure the effectiveness of a nation's consequence management using metrics, quantitative data assessment and progression over time." This fundamental objective drove the development of the functional and value hierarchies. These hierarchies are extremely important in that they establish the foundational construct of the CAM.

The first step in creating the value hierarchy is developing a functional hierarchy based on the fundamental objective in order to organize the critical aspects of the fundamental objective that the system must accomplish. The two most important functions supporting the fundament objective are "Develop Capacity" and "Provide Capability". Capacity is the extent to which a given capability can be performed, yielded, or withstood. It helps to determine “...how quickly the desired capabilities can be mobilised, how much capability is available, and for how long it can be deployed" (Moroney et al., 2007). Capability is the tangible ability to perform a function. It measures "...the ability to perform a function, i.e. the type, quality, and quantity of knowledge, skills, material support, and interoperability achieved" (Moroney et al., 2007).

The functional hierarchy expands into the value hierarchy through analysis of the functions into supporting objectives and corresponding value measures (see Figure 2). Under these two overarching categories, six objectives were created and 26 value measures were established to support the objectives. These value measures encompass all the areas that are required to assess for a nation's consequence management abilities.

Figure 2 Qualitative value hierarchy

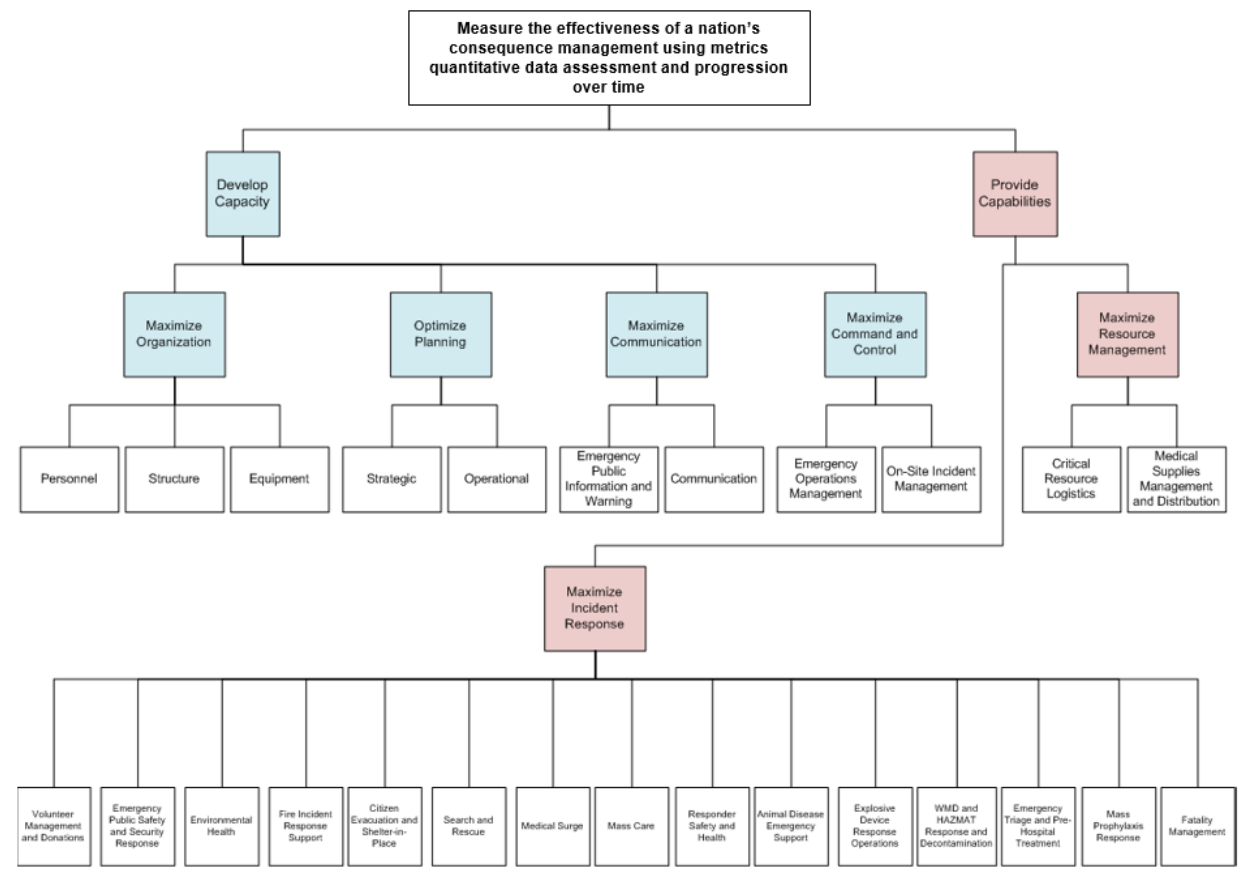


For the function "develop capacity", four objectives were identified as most critical: maximise organisation, optimise planning, maximise communication and maximise command and control. These four are identified and vetted by the stakeholder as the objectives that have the most effect on the function of developing capacity. In a similar way, for the second function, "provide capabilities", the most effective objectives are maximise incident response and maximise resource management. After the objectives of each function are defined and understood, value measures are identified for each of the objectives. For example, starting on the far left of the diagram, under the function "develop capacity", the objective "maximise organisation" has three value measures: personnel, structure and equipment (see Figure 2). The value measure "personnel" is defined as the body of persons employed by consequence management organisations. The intent with this value measure is to assess the personnel component of the organisation. Do you have the right number of people and are they trained/qualified in what is required in the consequence management field? A score is assessed based on how the nation performs under the criteria of the value measure. For this model, there are a total of 26 value measures. These 26 value measures are from the relevant consequence management critical areas identified by the stakeholder. Each value measure is defined with a scale to allow the data collector to easily and accurately classify the status of the nation in each category. Also, each value measure is weighted by the stakeholders according to their level of importance. After each value measure is scored, that score is then multiplied by the value measure normalised weight and the objective normalised weight that produces the score for that specific value measure. These individual scores are then summed together and divided by the ideal score to obtain the overall national score out of 100 .

The model is used to measure effectiveness and identify areas of weakness within a nation's consequence management program. The value measures are in a standard format so that users will go through a similar analysis every time the model is used. The combination of weighted value measures, objectives, and the scoring of each value measure provides an overall score. Nations will have a clear understanding of the areas that need improvement after they receive their evaluation. The end state of this model is the ability to provide nations with accurate feedback to determine where to focus resources and track progress over time.

\subsection{Weighting the value measures}

The importance of each value measure when compared to the other value measures varies. In order to establish a weighting system that takes into account the stakeholder values, a ranking method was introduced to rank and then assign relative weights to each value measure. In Figure 3, the stakeholder identified the relative importance of the value measures according to high, medium and low criteria. Figure 3 is a culmination of that ranking. Once a high, medium and low ranking was established, the value measures were prioritised within the ranking and then assigned relative weights to each value measure by the stakeholder. The weights were totalled and normalised and a weight was assigned to each value measure. This is also done for the objectives. 
Figure 3 Value measure weighting

\begin{tabular}{|c|c|c|c|c|c|}
\hline Value Measures & High & Medium & Low & Rank & Swing Weight \\
\hline Strategic & $x$ & & & 1 & 95 \\
\hline Operation & $x$ & & & 1 & 95 \\
\hline Structure & $x$ & & & 2 & 90 \\
\hline Personnel & $x$ & & & 3 & 86 \\
\hline Equipment & $x$ & & & 4 & 80 \\
\hline Emergency Operations Center Management & $x$ & & & 5 & 75 \\
\hline Communication & $x$ & & & 6 & 60 \\
\hline Emergency Public Information and Warning & $x$ & & & 7 & 60 \\
\hline MMD and HAZMAT Response and Decontamination & $x$ & & & 8 & 55 \\
\hline Emergency Triage and Prehospital Treatment & $x$ & & & 9 & 54 \\
\hline Responder Safety and Health & $x$ & & & 10 & 50 \\
\hline Fatality Management & & $x$ & & 1 & 49 \\
\hline Onsite Incident Management & & $x$ & & 2 & 47 \\
\hline Search and Rescue & & $x$ & & 3 & 45 \\
\hline Mass Care & & $x$ & & 4 & 43 \\
\hline Medical Surge & & $x$ & & 5 & 41 \\
\hline Mass Prophylaxis Response & & $x$ & & 6 & 39 \\
\hline Emergency Public Safety and Security Response & & $x$ & & 7 & 37 \\
\hline Environmental Health & & $x$ & & 8 & 35 \\
\hline Explosive Device Response Operations & & $x$ & & 9 & 33 \\
\hline Citizen Evacuation and Shelter-in-Place & & & $x$ & 1 & 30 \\
\hline Critical Reponse Logistics & & & $x$ & 2 & 27 \\
\hline Medical Supplies Management and Distribution & & & $x$ & 3 & 25 \\
\hline Animal Disease Emergency Support & & & $x$ & 4 & 23 \\
\hline Fire Incident Response Support & & & $x$ & 5 & 21 \\
\hline Volunteer Management and Donations & & & $x$ & 6 & 18 \\
\hline
\end{tabular}

\subsection{Model}

The model is an Excel-based scoring system where each value measure renders a score which is then weighted and finally summed and the end. In Figure 4 is an example of the value measure personnel and how it scores and is assigned a value to each of the scores. A qualitative description of the personnel category is assigned to each score $(0-5)$. A qualitative description of a score would be, for example, "All personnel receive formal training and pass test." If this score was a 2 , then the value of that score would receive a 50. The scorer would assign the appropriate score for each value measure based upon how the nation perform. If there are multiple personnel inputting data that is collected over a period of time, they begin by putting all of the data into the "Multiple Inputs" page of the model. If there is only one person collecting data for a nation, they enter the data directly into the "Input Scores" page of the model. The purpose of the "Multiple Inputs" page is to get an average score for each of the value measures so that the data collected during the engagement is not reliant on a single user's evaluation. If it is impossible to measure a certain metric for a nation it is possible to leave a value measure out of the model and it will still calculate correctly. 
Figure 4 Example value measure scale

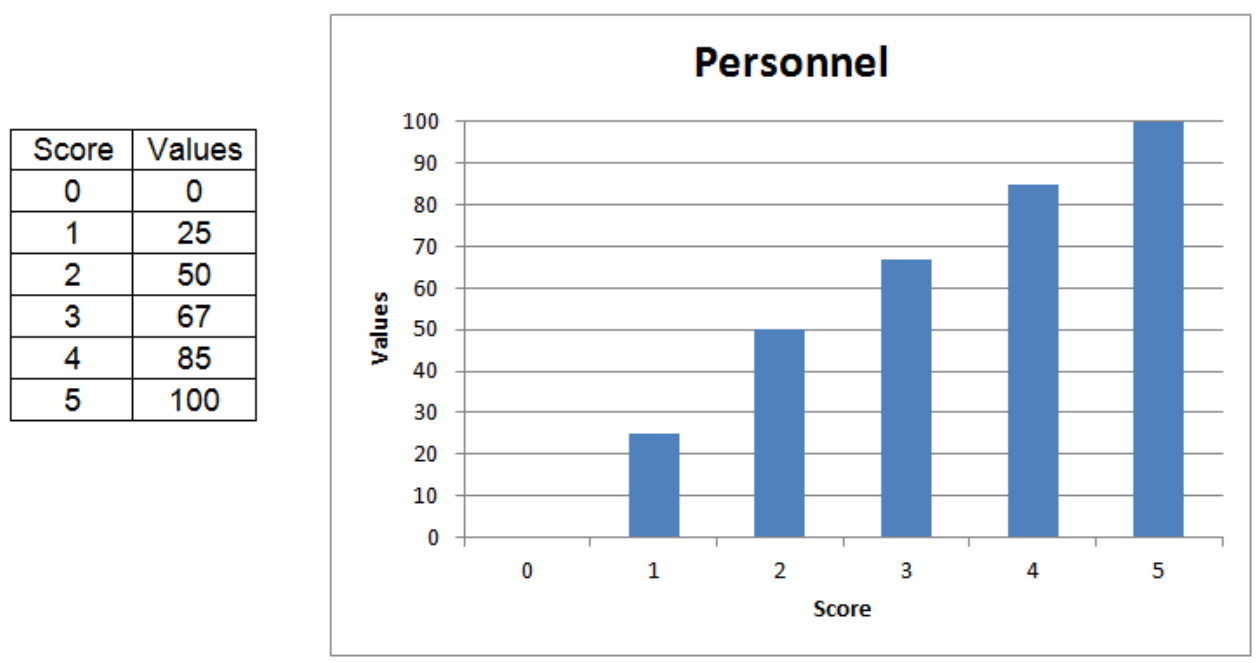

Figure 5 Bar chart, radar diagram, and overall nation score

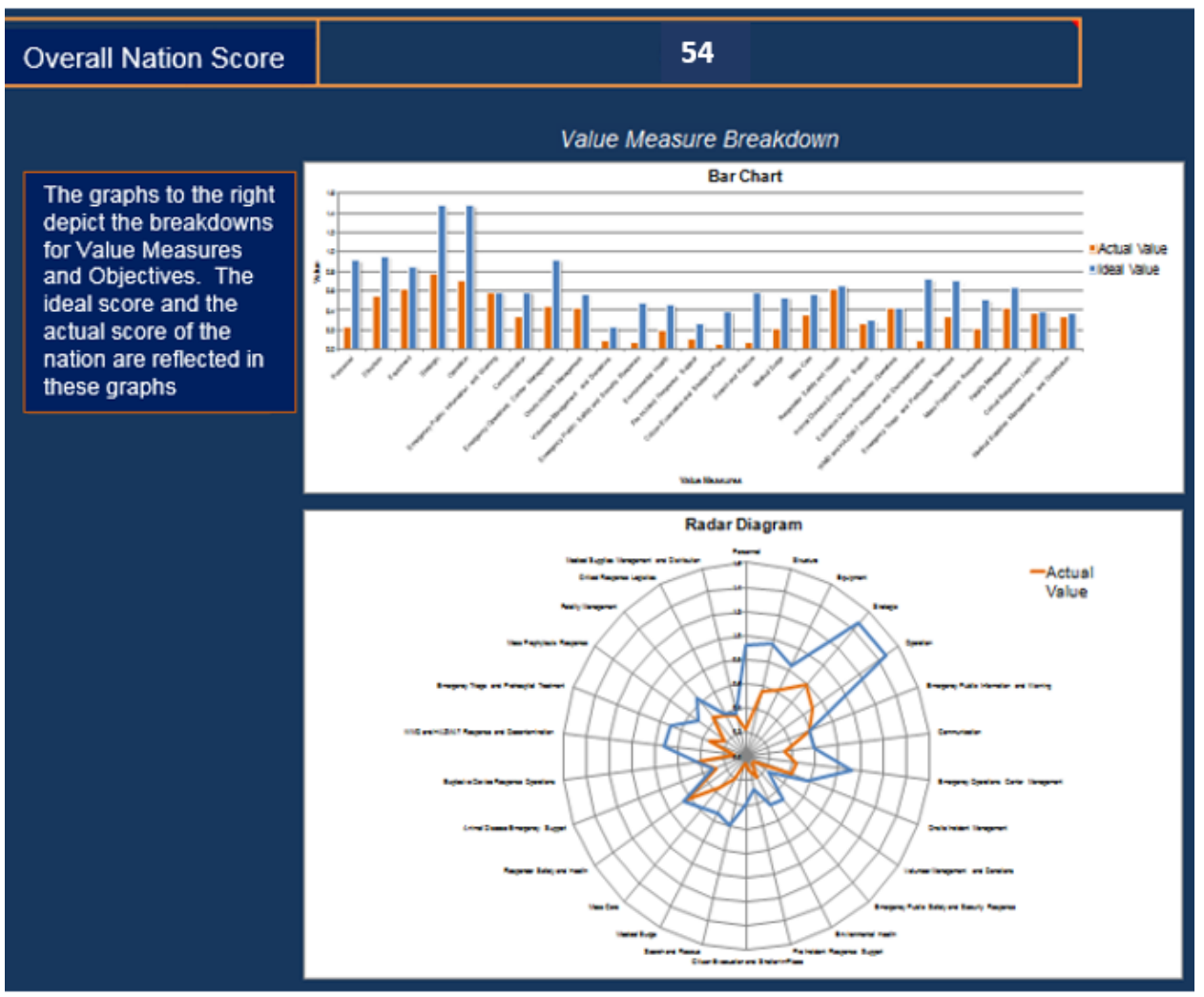


Once the data for all of the value measures is placed into the "Input Scores" page, the calculations run automatically and populate the weighted score for that value measure. The model converts the raw data score into the value from the value measure tables. That value is then multiplied by the associated normalised objective weight. That new value is then multiplied by the normalised weight of the individual value measure. Following these calculations, the individual values are summed together and divided by the ideal score to obtain the overall nation score out of 100 .

The evaluation page of the model displays the overall nation score. This score is measured on a 100 point scale and will give the user and stakeholders a summary of the nation's current consequence management capabilities and capacities (see Figure 5). The evaluation page also allows nation to locate deficient value measures and objectives. The bar chart and the radar diagrams display the actual nation score and compares that to the ideal score (the best a nation could have scored).

The archive page of the model contains the data of previous evaluations. This page serves as the database for the CAM. It contains the date of the evaluation, the score that the nation achieved, the individual value measure scores, and any user comments. This page allows nations to track the progress and compare their capabilities and capacities over time.

\section{Summary and conclusions}

The future seems to be directed toward increased catastrophic events both natural and induced. Nations will need to be prepared to respond and lessen the impact of consequences of these events. The CAM is a tool which can provide an opportunity to improve a nation's preparation. The CAM framework is used to measure, track, and identify areas of weakness within a nation's consequence management program. The goal of CAM is to maximise the capacity and capability of a nation and provide solid feedback on where they are lacking and may want to direct resources. Using the CAM, this goal can be achieved through the use of six objectives and 26 value measures that effectively capture the capacities and capabilities of a nation's consequence management profile. The objectives and value measures are in a standardised format so that users can go through a similar analysis every time the framework is used. The model can be used so that every metric can be examined each time so that nations develop a clearer understanding of the way ahead and how to improve. If it is impossible to measure a certain metric for a nation it is possible to leave a value measure out of the model and it will still calculate correctly.

In the future, creating a database and a more user-friendly input application will allow a nation to better track its progress. After each evaluation, a statistical analysis (to include propagation of error) can be completed to give a more in-depth analysis of the accuracy of the evaluation. Also, a database can be created in order to organise the evaluations over time and provide the nation with greater data to improve. Another useful tool is a collection system or application to increase usability and allow nation data collectors to easily input data into the model while in the field. 


\section{References}

Altay, N. and Green III, W.G. (2006) 'OR/MS research in disaster operations management', European Journal of Operational Research, pp.475-493.

Arms Control Association (2014) Chronology of U.S.-North Korean Nuclear and Missile Diplomacy. [Online] Available online at: http://www.armscontrol.org/factsheets/dprkchron (accessed on 26 June 2016).

Bennett, B.W. and Love, R.A. (2004) Initiatives and Challenges in Consequence Management after a WMD Attack, USAF Counter Proliferation Center, Maxwell Air Force Base.

Betts, R. (2012) 'The new threat of mass destruction', Foreign Affairs, pp.26-41.

Guh-Sapir, D. and Lechat, M.F. (1986) 'Information systems and needs assessment in natural disasters: an approach for better disaster relief management', Disasters, pp.161-240.

Moroney, J.D.P. et al. (2007) Building Partner Capabilities for Coalitiion Operations, Rand Corporation, Pittsburgh.

Parnell, G.S., Driscoll, P.J. and Henderson, D.L. (2010) Decision Making in Systems Engineering and Management, John Wiley and Sons, New York.

Taylor, S.R., Rowe, A.M. and Lewis, B.M. (1999) Consequence Management in Need of a Timeout, Center for Counterproliferation Research, National Defense University, Washington, DC. 